\title{
POROUS MEDIUM COMBUSTION: IGNITION, TEMPORAL EVOLUTION, AND PARAMETER DEPENDENCE
}

\author{
K. K. TAM
}

(Received 19 September 1989; revised 18 July 1990)

\begin{abstract}
A model for the combustion of a porous medium is considered for an infinite slab. The case of ignition by an initial temperature distribution is considered first. The influence of the initial data and parameters on the solution is inferred from the solution of a related ordinary differential equation. The case of ignition by heating on one side of the slab is then considered in the same manner.
\end{abstract}

\section{Introduction}

Recently, Norbury and Stuart [2] proposed a model for porous medium combustion. The governing equations are

$$
\begin{gathered}
\frac{\partial \sigma}{\partial t}=-\lambda r, \\
\mu \frac{\partial w}{\partial z}=u-w, \\
\sigma \frac{\partial u}{\partial t}=\frac{\partial}{\partial z}\left\{\left(d u^{3}+1\right) \frac{\partial u}{\partial z}\right\}+w-u+r, \\
\frac{\partial g}{\partial z}=-\frac{a r}{\mu},
\end{gathered}
$$

and

$$
r=H\left(\sigma-\sigma_{a}\right) H\left(u-u_{c}\right) \mu^{\frac{1}{2}} g w^{2}
$$

\footnotetext{
'Department of Mathematics and Statistics, McGi'l University, Montréal, Québec. (C) Copyright Australian Mathematical Society 1991, Serial-fee code 0334-2700/91
} 
Here $t$ and $z$ are the time and space variables; the nondimensionalised $\sigma$, $w, u$, are respectively the solid heat capacity, the gas temperature, and the solid temperature; $g$ is proportional to the product of oxygen concentration and gas temperature. The parameter $\lambda$ relates the amount of combustible solid present to the heat content of the solid; the parameter $\mu$ is proportional to the inlet gas mass flux; and the parameter $a$ represents the ratio of the rate of oxygen consumption to that of solid consumption. The parameter $d$ is a measure of the nonlinear radiation coefficient. The reaction rate $r$ involves two Heaviside functions with threshold parameters $\sigma_{a}$ and $u_{c}$, giving it a switching behavior. Travelling wave solutions to the above system has been investigated by Norbury and Stuart [3], [4] and by Tam [5].

In this note, we pose the following question. Suppose a porous slab of unit width undergoes combustion, what can be said about the qualitative aspects of its temporal evolution, as well as the parameter dependence? It is clear that an analytic solution to the above system is not attainable. The answer to the question would likely rely on further simplifications which retain the salient features of the system, but renders it manageable. In the next two sections, we consider ignition resulting from an initial temperature distribution, and in Section 4, we consider ignition resulting from heating on one side of the slab.

\section{Formulation and approximation}

Let the porous slab occupy $0 \leq z \leq 1$. We impose the following conditions on the system (1-1) to (1-4):

$$
\begin{aligned}
& \sigma(x, 0)=\sigma_{s}, \quad \sigma(x, \infty)=\sigma_{a}<\sigma_{s}, \\
& u(0, t)=0, \quad u(1, t)=0, \quad u(z, 0)=u_{0}(z), \\
& w(0, t)=0, \quad g(0, t)=g_{a} .
\end{aligned}
$$

Here we have normalised $u$ and $w$ so that the ambient temperature is taken as zero. Of the two parameters $\sigma_{a}$ and $u_{c}$ in (1-5), $\sigma_{a}$ is the solid heat capacity of the ash (burnt medium) and can be considered well defined. The threshold temperature $u_{c}$ for reaction to start is not so well defined. Moreover, (1-2) shows that if $u>w, w$ increases (with $z$ ). Thus, if $u$ is large, $w$ will be large also. For this reason, we simplify (1-5) by removing $H\left(u-u_{c}\right)$, so that the reaction rate is no longer governed explicitly by the solid temperature. Writing $\chi=\sigma-\sigma_{a}$ and $\chi_{0}=\sigma_{s}-\sigma_{a}$, and noting that $r$ is non-negative, we recognise from $(1-1)$ that for $0 \leq z \leq 1,0<t<\infty$, we have $0<\chi<\chi_{0}$. Now, we recognise that the switching factor $H\left(\sigma-\sigma_{a}\right)$ is used so that the expression $r$ has a manageable structure, but it is also clear that in most decay processes, the time rate of decay is affected by the 
magnitude of the quantity under consideration. In the present situation, retaining the factor $\left(\sigma-\sigma_{a}\right)$ in $r$ does not lead to much additional difficulty. We therefore replace $H\left(\sigma-\sigma_{a}\right)$ by $\left(\sigma-\sigma_{a}\right)$ and take

$$
r=\left(\sigma-\sigma_{a}\right) \mu^{\frac{1}{2}} g w^{2} \text {. }
$$

We convert (1-1), (1-2) and (1-4) into the corresponding integral relations

$$
\begin{aligned}
& \chi=\chi_{0} \exp \left\{-\lambda \mu^{\frac{1}{2}} \int_{0}^{t} g w^{2} d \tau\right\}, \\
& g=g_{a} \exp \left\{-a \mu^{-\frac{1}{2}} \int_{0}^{z} \chi w^{2} d s\right\},
\end{aligned}
$$

and

$$
w=\frac{1}{\mu} e^{-\frac{z}{\mu}} \int_{0}^{z} u e^{\frac{s}{\mu}} d s .
$$

To proceed further, we observe that since $0<\chi<\chi_{0}$, it is clear that

$$
g_{a} \exp \left\{-a \mu^{1 / 2} \chi_{0} \int_{0}^{2} w^{2} d s\right\}<g<g_{a} .
$$

Consistent with our objective simplification while retaining the salient feature of the system, and in the spirit of modified Oseen linearisation [1], we adopt the approximation

$$
g \doteq g_{a} \exp \left\{-a \mu^{1 / 2} \chi^{*} \int_{0}^{z} w^{2} d s\right\}
$$

where $\chi^{*}=\chi_{0} / 2$. The advantage is that $g$ is now determined by $w$, which is given in terms of $u$. If we now substitute (2-2), (2-4) in (1-3), we see that it is an equation involving only the unknown $u$, albeit with integrals and derivatives thereof. We suppose that the problem under consideration admits a unique solution $u(z, t)$, which is positive for $0<z<1,0<t<\infty$. Hence, we can consider an expansion of $u$ in terms of the eigenfunctions $\phi_{n}(z)$ defined by

$$
\phi_{n}=-\gamma_{n}^{2} \phi_{n}, \quad \phi_{n}(0)=\phi_{n}(1)=0,
$$

where $\gamma_{n}^{2}$ is the eigenvalue corresponding to $\phi_{n}$. Indeed, we have $\gamma_{n}=n \pi$ and $\phi_{n}=\sqrt{2} \sin n \pi z$. We observe that $\phi_{1}(z)$ is positive in $0<z<1$, while all higher eigenfunctions change sign in $0<z<1$. In the expression

$$
u(z, t)=\sum_{n=1}^{\infty} A_{n}(t) \phi_{n}(z),
$$


$u>0$ implies that $A_{1}(t) \phi_{1}(z)$ must dominate, (otherwise, $u$ may become negative), and $A_{1}(t)$ must be positive for $0<t<\infty$. If $u$ is large, it implies $A_{1}$ is large, and conversely. If $u$ is small, it implies $A_{1}$ is small, and conversely. Accepting the validity of this observation, we see that the temporal evolution of $u$ can be inferred from the temporal evolution of $A_{1}(t)$.

If we now take $u(z, t) \sim A_{1}(t) \phi(z)$, and consider only the point $z=\frac{1}{2}$, at which $\phi_{1}$ acquires its maximum value, we obtain an ordinary differential equation. We propose that this is an approximate equation governing $A_{1}(t)$. In what follows, we omit the subscript in $A_{1}(t)$. We have

$$
\begin{aligned}
w\left(\frac{1}{2}, t\right) & \doteq \frac{1}{\mu} e^{-\frac{1}{2 \mu}} A(t) \int_{0}^{\frac{1}{2}} \phi_{1}(s) e^{\frac{s}{\mu}} d s \equiv W\left(\frac{1}{2}\right) A(t) \\
\sigma\left(\frac{1}{2}, t\right) & =\sigma_{a}+\chi\left(\frac{1}{2}, t\right) \\
& \doteq \sigma_{a}+\chi_{0} \exp \left\{-\lambda \mu^{\frac{1}{2}} W^{2}\left(\frac{1}{2}\right) \int_{0}^{t} g A^{2} d \tau\right\} \\
g\left(\frac{1}{2}, t\right) & \doteq g_{a} \exp \left\{-a \mu^{-\frac{1}{2}} \chi^{*} A^{2}(t) \int_{0}^{\frac{1}{2}} W^{2}(s) d s\right\} \\
& \equiv g_{a} \exp \left\{-G A^{2}(t)\right\}
\end{aligned}
$$

where we have used $G$ and $W$ for simplicity, and $G$ and $W$ are defined by (2-6) and (2-8). It then follows from (1-3) that

$$
\begin{aligned}
\sigma\left(\frac{1}{2}, t\right) \phi\left(\frac{1}{2}\right) \frac{d A}{d t}= & -\left\{\left(\gamma_{1}^{2}+1\right) \phi_{1}\left(\frac{1}{2}\right)-W\left(\frac{1}{2}\right)\right\} A-d \gamma_{1}^{2} \phi_{1}^{4}\left(\frac{1}{2}\right) A^{4} \\
& +\mu^{\frac{1}{2}} g_{a} W^{2}\left(\frac{1}{2}\right) \chi\left(\frac{1}{2}, t\right) A^{2} \exp \left(-G A^{2}\right) .
\end{aligned}
$$

For simplicity, we write the right side of (2-9) as $-K_{1} A-K_{2} A^{4}+K_{3} A^{2} e^{-G A^{2}}$. Clearly, $d A / d t$ vanishes at $A=0$ and when $A$ is a solution of the transcendental equation

$$
K_{1}+K_{2} A^{3}=K_{3}(t) A \exp \left(-G A^{2}\right) .
$$

While (2.10) cannot be solved analytically, its behavior is readily discernible. We discuss this in the following section.

\section{Ignition, temporal evolution and parameter dependence}

The behavior of the solution to (2.10) can be examined with the help of Figure 1. 


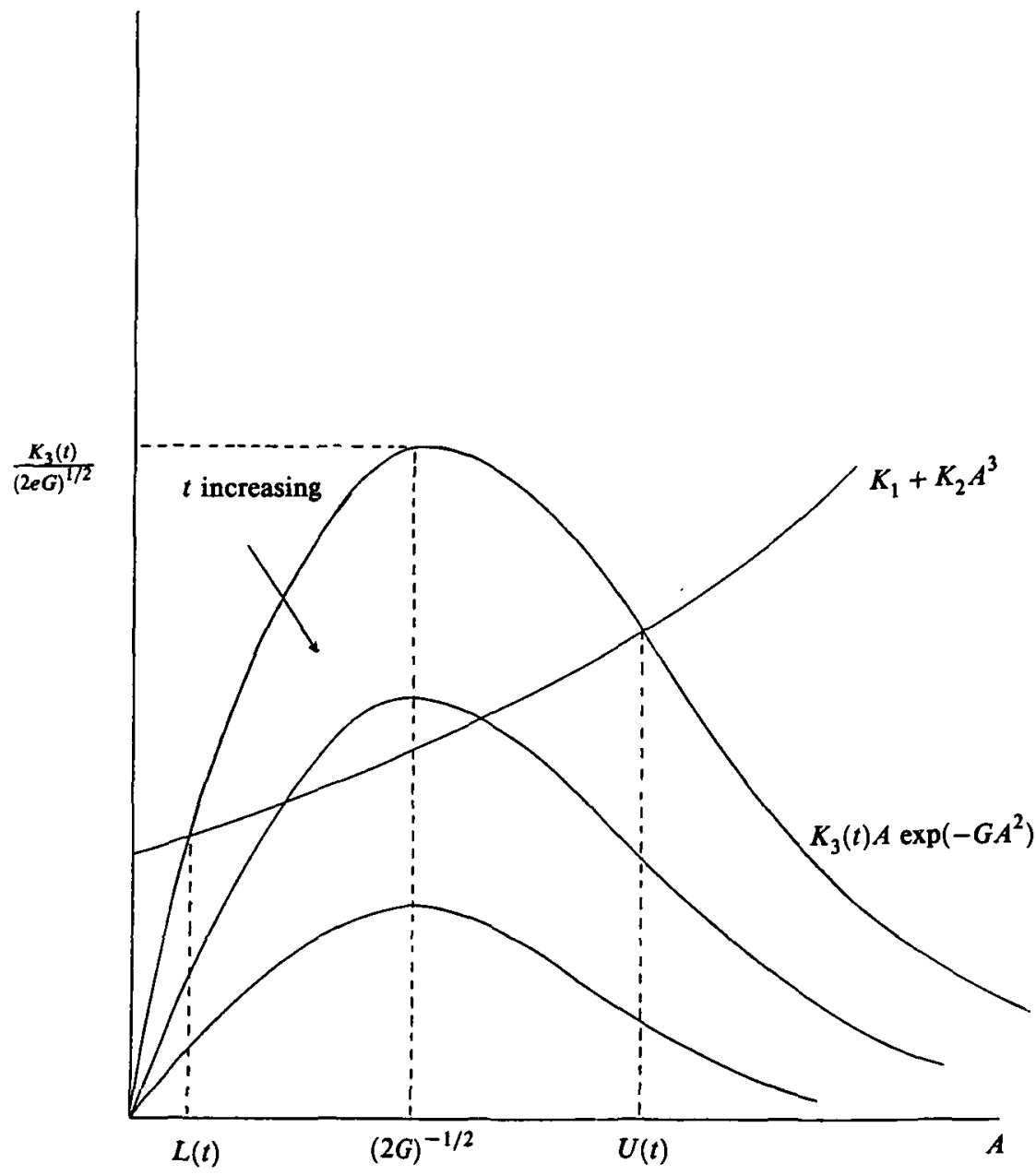

Figure 1. Schematic interpretation of (2-9).

The function $K_{3}(t) A \exp \left(-G A^{2}\right)$ has a relative maximum at $A=(2 G)^{-\frac{1}{2}}$, where its value is $K_{3}(t)(2 e G)^{-\frac{1}{2}}$. We make the following observations:

1) If

$$
K_{3}(0)(2 e G)^{-\frac{1}{2}}<K_{1}+K_{2}(2 G)^{-\frac{2}{3}}
$$

then it is clear that $d A / d t>0$ for whatever value of $A(0)$, and $A$ decays to zero as $t$ tends to infinity. Thus, the process is essentially one of diffusion. In terms of the original parameters, the inequality 
$(3-1)$ is

$$
\begin{aligned}
\mu^{\frac{1}{2}} g_{a} W^{2}\left(\frac{1}{2}\right) \chi\left(\frac{1}{2}, 0\right)< & \left\{\left(\gamma_{1}^{2}\right) \phi_{1}\left(\frac{1}{2}\right)-W\left(\frac{1}{2}\right)\right\} \\
& +\gamma_{1}^{2} d \phi_{1}^{4}\left(\frac{1}{2}\right)(2 G)^{-\frac{2}{3}}
\end{aligned}
$$

Using $\gamma_{1}=\pi, \phi_{1}=\sqrt{2} \sin \pi z$, we have

$$
\begin{gathered}
W\left(\frac{1}{2}\right)=\frac{\sqrt{2}\left[1+\mu \pi \exp \left(-\frac{1}{2 \mu}\right)\right]}{\left(1+\mu^{2} \pi^{2}\right)}, \\
G=\frac{a \chi^{*}}{2 \mu^{\frac{1}{2}}\left(1+\mu^{2} \pi^{2}\right)^{2}} \\
\quad \times\left\{1+2 \mu^{3} \pi^{4}\left(1-e^{-\frac{1}{\mu}}\right)+\mu^{2} \pi^{4}-8 \mu^{2} \pi^{2} e^{-\frac{1}{2 \mu}}-4 \mu \pi\right\} .
\end{gathered}
$$

With $\chi\left(\frac{1}{2}, 0\right)=\sigma_{s}-\sigma_{a}$, and considering the case of $\mu \rightarrow 0$, where $W \rightarrow \sqrt{2}$, we see that combustion does not occur if

$$
2 \mu^{\frac{1}{2}} g_{a}\left(\sigma_{s}-\sigma_{a}\right)<\pi^{2} \text {. }
$$

2) If $k_{3}(0)(2 e G)^{-\frac{1}{2}}>K_{1}+K_{2}(2 G)^{-\frac{3}{2}}$, then (2-10) admits two solutions, say $L(0)$ and $U(0)$, as depicted in Figure 1. If $A(0)<L(0), A$ decays to zero. If $L(0)<A(0)<U(0) A$ increases towards $U(0)$; but as $t$ increases $U(t)$ decreases. When $A(t)$ reaches $U(t), A(t)$ acquires its maximum value and from then on $d A / d t<0$, so that $A$ decays to zero as $t$ tends to infinity. If $A(0)>U(0), d A / d t<0$, and so $A$ decays to zero as $t$ tends to infinity. The case $L(0)<$ $A(0)<U(0)$ exhibits the typical combustions phenomenon. If the initial temperature $A(0)$ is higher then the threshold temperature $L(0), A$ starts to increase (ignition). Having reached a maximum temperature, $A$ decreases as a result of the depletion of the combustion medium.

3) To obtain an estimate of $U(0)$, we first observe that the curve $K_{3}(0) A \exp \left(-G A^{2}\right)$ lies below the curve

$$
K_{3}(0)(2 e G)^{-\frac{1}{2}} \exp \left\{-G\left[A-(2 G)^{-\frac{1}{2}}\right]^{2}\right\}
$$

for $A>(2 G)^{-\frac{1}{2}}$. Thus, $V(0)$ must lie between $(2 G)^{-\frac{1}{2}}$, and the intersection of $K_{1}$ and $K_{3}(0)(2 e G)^{-\frac{1}{2}} \exp \left\{-G\left[A-(2 G)^{-\frac{1}{2}}\right]^{2}\right\}$, 
which is

$$
\frac{1}{(2 G)^{\frac{1}{2}}}+\left[\frac{1}{G} \log \left\{\frac{K_{3}(0)}{\left(K_{1}(2 e G)^{1 / 2}\right.}\right\}\right]^{2} .
$$

The true value of $U(0)$ is closer to the expression in (3-4) if $d$ is small, and closer to $(2 G)^{-\frac{1}{2}}$ if $d$ is large. In terms of the original parameters, and considering $\mu \rightarrow 0$, we have

$$
\frac{\mu^{\frac{1}{4}}}{\left(a \chi^{*}\right)^{\frac{1}{2}}}<U(0)<\frac{\mu^{\frac{1}{4}}}{\left(a \chi^{*}\right)^{\frac{1}{2}}}\left\{\log \frac{2 \mu^{\frac{3}{4}} g_{a} \chi\left(\frac{1}{2}, 0\right)}{\pi^{2} \sqrt{a \chi^{*} e}}\right\} .
$$

For $\frac{K_{3}(0)}{K_{1}(2 e G)^{1 / 2}}$ ranging from 10 to 10000 , the value of the term

$$
\left\{\log \left[K_{3}(0) / K_{1}(2 e G)^{\frac{1}{2}}\right]\right\}^{\frac{1}{2}}
$$

ranges from 1.5 and 2.6. Thus, it is clear that $U(0)=O\left(\mu^{\frac{1}{4}} / \sqrt{a \chi^{*}}\right)$. Since $\mu$ is small, $a$ must also be small for $U(0)$ to be large.

4) An estimate of $L(0)$ is given by the intersection of $K_{1}+K_{2} A^{3}$ and $K_{3}(0) A$. While the solution of this cubic equation can be obtained explicitly, we note simply that for $d$ small, i.e., $K_{2}$ small, we have

$$
L(0)=K_{1} / K_{3}(0) \text {. }
$$

For $\mu \rightarrow 0$, we have

$$
\frac{K_{1}}{K_{3}(0)}=\frac{\pi^{2}}{\sqrt{2 \mu} g_{a} \chi_{0}} .
$$

Thus, the threshold value is inversely proportional to $g_{a} \chi_{0}$.

5) To bring (2), (3) and (4) into focus, suppose the initial condition on $u$ is $u(z, 0)=A(0) \phi_{1}(z)$. Then for $\mu \rightarrow 0$, the typical combustion phenomenon is exhibited if

$$
\frac{7}{g_{a} \chi_{0} \sqrt{\mu}}<A(0)<5 \frac{\mu^{1 / 4}}{\sqrt{a \chi_{0}}} .
$$

Clearly, the parameters $a, g_{a}, \chi_{0}$ must satisfy the inequality

$$
\frac{1.4}{g_{a}} \sqrt{\frac{a}{\chi_{0}}}<\mu^{3 / 4}
$$

6) The parameter $\lambda$ governs the rate that $\chi$ decays. With reference to Figure 1, a large $\lambda$ implies that the bell-shaped curve drops rapidly, causing a rapid burn-out of the medium. This result agrees with that obtained in Norbury and Stuart [4]. 


\section{Boundary ignition}

In this section, we let the initial temperature of the medium be zero, but consider the possibility of ignition when the temperature on one face of the slab is raised to a constant value. Specifically, we consider the initial and boundary conditions on $u$ as

$$
u(0, t)=y>0, \quad u(1, t)=0,
$$

and

$$
u(z, 0)=0
$$

All other conditions remain unchanged as in Section 2. Our interest is now on the dependence of the solution on $h$. To simplify the situation, we let $d=0$.

We write $u=\theta+U$, and ask that $U$ satisfies the equation

$$
\sigma U_{t}=U_{z z}-U
$$

and the conditions

$$
U(z, 0)=0 ; \quad U(0, t)=h, U(1, t)=0
$$

Clearly, $U$ reaches the steady state

$$
U_{s}=\frac{h}{\sinh 1} \sinh (1-z) .
$$

The expression for $\chi$ and $g$ can be written again as in (2-2) and (2-5), but now $w$ becomes

$$
w=\mu^{-1} e^{-\frac{z}{u}} \int_{0}^{z}(\theta+u) e^{\frac{s}{\mu}} d s
$$

while $\theta$ is governed by equation (1-3) with $u$ replaced by $\theta$ and $d=0$. Although $U$ cannot be determined analytically, it is clear that $U<U_{s}$ for $0<z<1, t>\infty$; its effect on $w$ can be assessed through its steady state $U_{s}$. Again, using the heuristic argument that the behaviour of $\theta$ parallels that of its first Fourier component $B(t) \phi_{1}(z)$, where $\phi_{1}$ is the first eigenfunction as 
in Section 2, we obtain, in a similar manner, the following expressions:

$$
\begin{aligned}
w(z, t) \doteq & \frac{\sqrt{2} B(t)}{1+\mu^{2} \pi^{2}}\left\{\mu \pi e^{-\frac{3}{\mu}}+\sin \pi z-\mu \pi \cos \pi z\right\} \\
& +\frac{h}{2 \sinh 1}\left\{\frac{e\left(e^{-z}-e^{-\frac{z}{\mu}}\right)}{1-\mu}-\frac{e^{z}-e^{-\frac{z}{\mu}}}{e(1+\mu)}\right\} \\
\equiv & W(z) B(t)+w_{h}(z), \\
\sigma\left(\frac{1}{2}, t\right)= & \sigma_{a}+\chi\left(\frac{1}{2}, t\right) \\
\doteq & \sigma_{a}+\chi_{0} \exp \left\{-\lambda \mu^{-\frac{1}{2}}\left[W^{2}\left(\frac{1}{2}\right) \int_{0}^{t} g B^{2} d r+2 W\left(\frac{1}{2}\right) W_{h}\left(\frac{1}{2}\right)\right.\right. \\
g\left(\frac{1}{2}, t\right) \doteq & g_{a} \exp \left\{-a \mu^{-\frac{1}{2}} \chi^{*}\left[B^{2}(t) \int_{0}^{\frac{1}{2}} W^{2}(s) d s\right.\right. \\
\quad & \left.\left.\quad 2 B(t) \int_{0}^{\frac{1}{2}} W(s) W_{h}(s) d s+\int_{0}^{2}\left(\frac{1}{2}\right) \int_{0}^{t} g d \tau\right]\right\} \\
\equiv & g_{a} \exp \left\{\left(G_{1} B^{2}(t)+G_{2}(s) d s\right\}\right\}
\end{aligned}
$$

It then follows from $(1-3)$ that

$$
\begin{aligned}
\sigma\left(\frac{1}{2}, t\right) \phi\left(\frac{1}{2}\right) \frac{d B}{d t}=-\left\{\left(\gamma_{1}^{2}+1\right) \phi_{1}\left(\frac{1}{2}\right)-W\left(\frac{1}{2}\right)\right\} B+W_{h}\left(\frac{1}{2}\right) \\
+\mu^{\frac{1}{2}} g_{a} \chi\left(\frac{1}{2}, t\right)\left\{W^{2}\left(\frac{1}{2}\right) B^{2}+2 W\left(\frac{1}{2}\right) W_{h}\left(\frac{1}{2}\right) B+W_{h}^{2}\left(\frac{1}{2}\right)\right\} \\
\quad \times \exp \left\{-G_{1} B^{2}-G_{2} B-G_{3}\right\} \\
=-R_{1}+R_{2}+R_{3} .
\end{aligned}
$$

The behaviour of the solution to (4-8) can be examined with the help of Figure 2. The right side of (4-8) has a unique zero $B_{0}(t)$ which is a decreasing function of $t$, tending to

$$
B(\infty)=\frac{W_{h}\left(\frac{1}{2}\right)}{\left(\gamma_{1}^{2}+1\right) \phi_{1}\left(\frac{1}{2}\right)-W\left(\frac{1}{2}\right)},
$$

as $t$ tends to infinity. As $t$ increases from zero, $B$ increases to $B_{0}(t)$, and then decreases to $B(\infty)$. If $B_{0}(0)$ is much greater than $B(\infty)$, which 


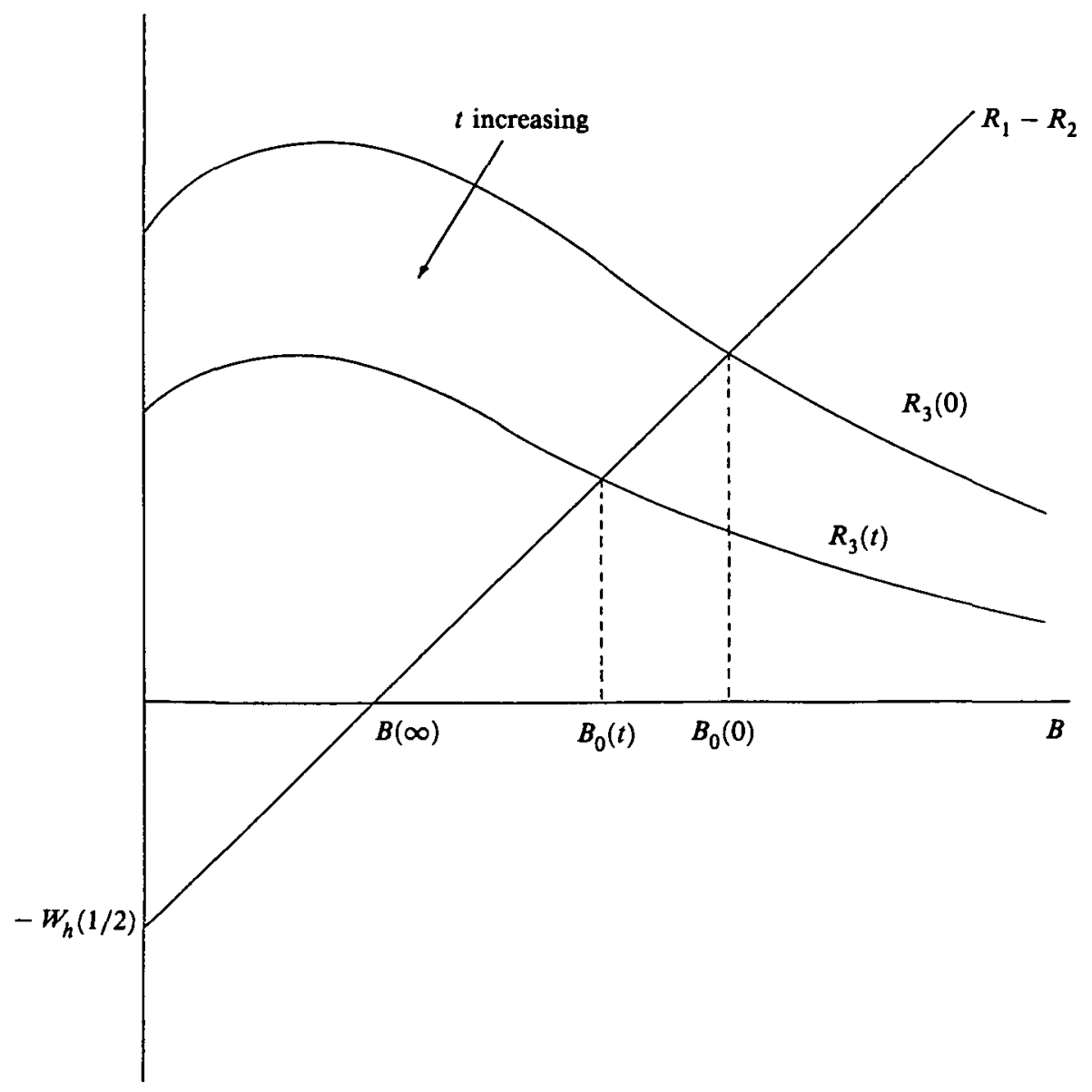

Figure 2. Schematic interpretation of (4-8).

implies a large value of $g_{a} \chi\left(\frac{1}{2}, 0\right) W_{h}^{2}\left(\frac{1}{2}\right)$, and a small value of $a \mu^{-\frac{1}{2}}$, we have ignition. However, if $B_{0}(0)$ is close to $B(\infty)$, the process is essentially one of diffusion.

\section{Concluding remarks}

For the initial-value problem with homogeneous boundary conditions, we use the heuristic argument that $u(z, t)$ and the first term of its Fourier decomposition $A_{1}(t) \phi_{1}(z)$ must have parallel behavior to obtain an equation which we believe is an approximate governing equation for $A_{1}(t)$. The threshold value, temporal evolution, and parameter dependence of $A_{1}(t)$ are 
then obtained in a simple manner. We expect that if $u(z, 0)$ does not deviate substantially from $\phi_{1}(z)$, the heuristic argument is correct and hence the question of ignition, temporal evolution and parameter dependence for $u(z, t)$ can be inferred. This study also suggests that the system of model equations does indeed contain the features of the combustion process.

For the case of boundary ignition, we separate the problem into two parts. One of which has a simple steady state, and the other is dealt with using the same heuristic argument. The dependence of $u(z, t)$ on boundary data and parameters can again be inferred.

\section{Acknowledgement}

This research was supported by the Natural Science and Engineering Research Council of Canada.

\section{References}

[1] G. F. Carrier, "Analytic approximation techniques in applied mathematics", SIAM J. Appl. Math. 13 (1965) 68-95.

[2] J. Norbury and A. M. Stuart, "A model for porous medium combustion", Q.J. Mech. Appl. Math. 42 (1989) 154-178.

[3] $\_$, "Travelling combustion waves in a porous medium Part I.-existence," SIAM J. Appl. Math. 48 (1988) 155-169.

[4] _ Travelling combustion waves in a porous medium Part II.-stability," SIAM J. Appl. Math. 48 (1988) 374-392.

[5] K. K. Tam, "Travelling wave solutions for combustion in a porous medium," Studies in Appl. Math. (1989) 249-262. 